\title{
Quasi-periodic Variations in the Hard X-ray Emission of a Large Arcade Flare
}

\author{
J. Jakimiec $\cdot$ M. Tomczak
}

Received: 26 June 2012 / Accepted: 7 March 2013 / Published online: 9 April 2013

(C) The Author(s) 2013. This article is published with open access at Springerlink.com

\begin{abstract}
We investigated the quasi-periodic oscillations of the hard X-ray (HXR) emission of the large flare of 2 November 1991 using HXR light curves and soft X-ray and HXR images recorded with the Yohkoh X-ray telescopes. We analysed these observations and report five main results: i) The observations confirm that electrons are accelerated in oscillating magnetic traps that are contained within the cusp magnetic structure. ii) The chromospheric upflow increases the density within the magnetic traps, which in turn together with the higher amplitude of the trap oscillations increases the amplitude of the HXR pulses. iii) This increase stops when the density inside the traps increases progressively and inhibits the acceleration of electrons. iv) The model of oscillating magnetic traps is able to explain the time variation of the electron precipitation, the strong asymmetry in the precipitation of the accelerated electrons, and the systematic differences in the precipitation of 15 and $25 \mathrm{keV}$ electrons. v) We have obtained direct observational evidence that strong HXR pulses are the result of the inflow into the accelerated volume of dense plasma from chromospheric evaporation.
\end{abstract}

Keywords Energetic particles · Flares $\cdot$ Impulsive phase $\cdot$ Oscillations

\section{Introduction}

In the hard X-ray (HXR) emission of many flares quasi-periodic variations were observed (Lipa, 1978; see also the review of Nakariakov and Melnikov (2009) and references therein).

In our previous papers (Jakimiec and Tomczak, 2010 (Paper I), 2012 (Paper II)) we investigated flares with periods $P=10-60 \mathrm{~s}$, but in Paper I we have found three flares with periods $P>120 \mathrm{~s}$. They turned out to be large arcade flares. Investigating the quasi-periodic

\footnotetext{
J. Jakimiec · M. Tomczak ( $\varangle)$

Astronomical Institute, University of Wrocław, ul. Kopernika 11, 51-622 Wrocław, Poland

e-mail: tomczak@astro.uni.wroc.pl

J. Jakimiec

e-mail: jjakim@ astro.uni.wroc.pl
} 
Figure 1 Soft X-ray image of the 30 September 1998 flare (intensity scale in colour). The isocontours show the HXR $14-23 \mathrm{keV}$ intensity. The solid line shows the solar limb and the dashed lines are on the solar disc.

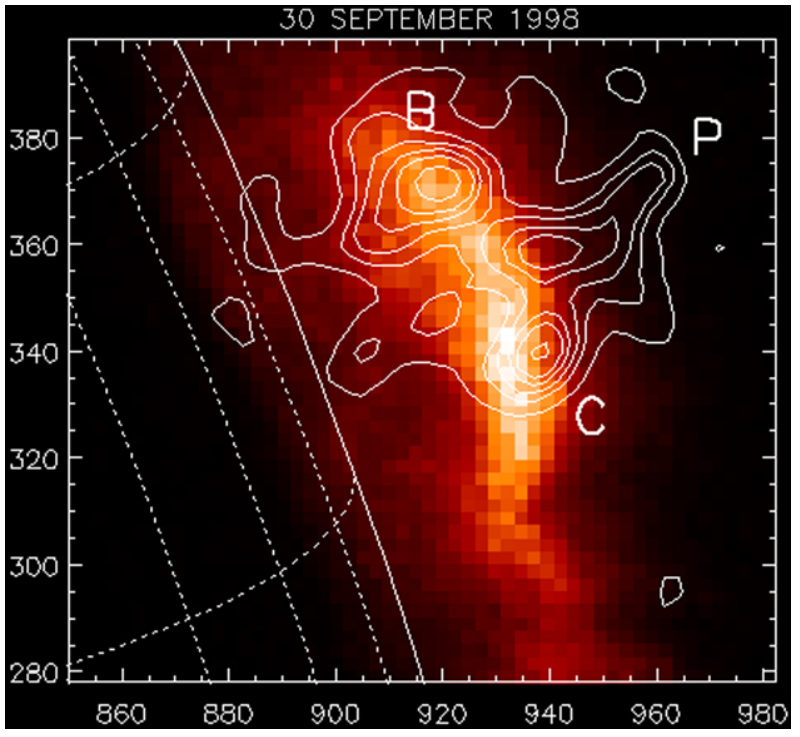

oscillations in these large flares is very important because their large sizes allow us to investigate the structure of the oscillation volume more comprehensively. Unfortunately, appropriate observations of the X-ray oscillations in such large flares are very rare. In the present paper we investigate an interesting example of such a large flare, that of 2 November 1991. Section 2 contains the analysis of observations and Sections 3 and 4 present the discussion and summary.

In Figure 1 we reproduce the X-ray image of the large flare of 30 September 1998 (see Paper I), which will be helpful in our analysis of the 2 November 1991 flare. In the colour SXR image a long arcade channel is visible. In the HXR image (isocontours) a triangular ("cusp") structure is seen above the arcade channel. The strong HXR sources B and C are located at the places where the cusp structure joins the arcade channel. This suggests that there is magnetic connection between the cusp and the channel at $\mathrm{B}$ and $\mathrm{C}$, which allows the electrons accelerated in the cusp to penetrate into the dense plasma of the channel and to generate the enhanced HXR emission there.

\section{Observations and Analysis}

We here investigate a large arcade flare that occurred at the western limb on 2 November 1991. It was a long-duration event (LDE) of GOES class M4.8. The soft X-ray emission had begun to rise at 15:32 UT and reached its maximum at about 17:00 UT (see the GOES light curves in Figure 2), i.e. it was of "slow-LDE" type according to Hudson and McKenzie (2000). The H $\alpha$ emission began at the northern footpoint of the flaring loop at 16:19 UT and was observed until 18:10 UT (see Solar-Geophysical Data No. 573/II).

The hard X-ray light curves recorded by the Yohkoh Hard X-ray Telescope HXT (Kosugi et al., 1991) and the Compton Gamma Ray Observatory Burst and Transient Source Experiment, BATSE (Fishman et al., 1992) are shown in Figures 3 and 4a. The nominal energy range of the BATSE observations is $h v>25 \mathrm{keV}$. The comparison of the light curves in Figures 3 and 4 shows that the strong peak at about 16:34 UT is more dominant in the BATSE 
Figure 2 The standard GOES light curves for the flare of 2 November 1991 (upper curve $1-8 \AA$ range, lower curve $0.5-4 \AA$ range). The hatched areas show the Yohkoh satellite nights.

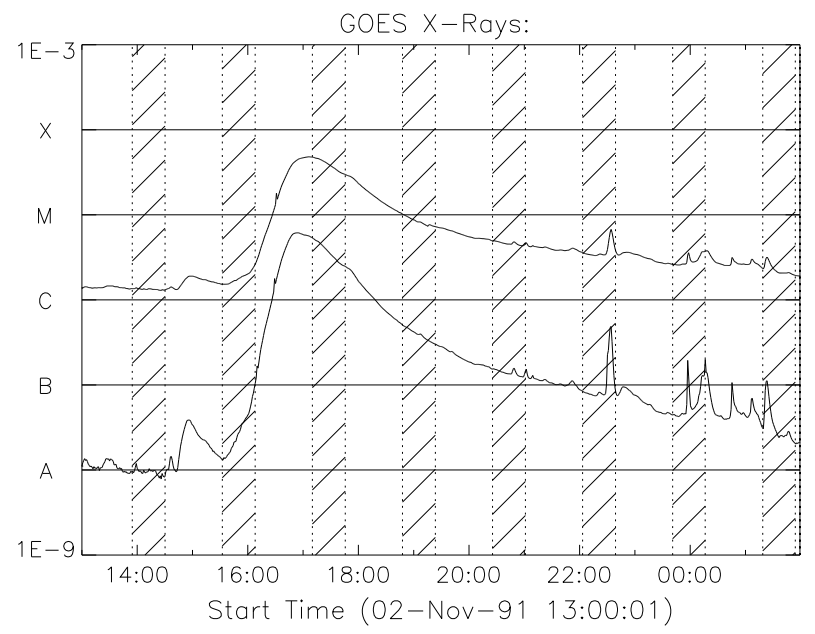

Table 1 Parameters of the quasi-periodic oscillations of the 2 November 1991 flare.

\begin{tabular}{|c|c|c|c|c|c|}
\hline \multicolumn{2}{|c|}{$\begin{array}{l}\text { During impulsive phase rise } \\
16: 20-16: 30 \text { UT }\end{array}$} & \multicolumn{2}{|c|}{$\begin{array}{l}\text { Maximum of impulsive phase } \\
16: 30-16: 36 \text { UT }\end{array}$} & \multicolumn{2}{|c|}{$\begin{array}{l}\text { After impulsive phase } \\
\text { 16:38-16:49 UT }\end{array}$} \\
\hline$P[\mathrm{~s}]$ & $\operatorname{Amp} S$ & $P[\mathrm{~s}]$ & $\operatorname{Amp} S$ & $P[\mathrm{~s}]$ & Amp $S$ \\
\hline $129 \pm 3$ & 0.94 & 164 & 0.67 & $115 \pm 10$ & 0.38 \\
\hline
\end{tabular}

$P$ is the mean time-interval between successive HXR peaks, and Amp $S$ is the mean value of the full amplitude of the $S$-function measured for individual HXR peaks.

observations than in the Yohkoh $23-33 \mathrm{keV}$ energy range. We have compared the amplitude ratio of the strong and weak peaks $(16: 21-16: 30 \mathrm{UT})$ in these sequences and found that the BATSE ratio agrees well with the Yohkoh $33-53 \mathrm{keV}$ ratio, which indicates that the actual energy range of the BATSE observations was $h v>33 \mathrm{keV}$.

\subsection{Analysis of the Quasi-periodicity of the HXR Pulses}

To separate the HXR pulses from the smooth HXR rise we calculated the normalised time series $S(t)$ (see Paper II),

$$
S(t)=\frac{F(t)-\hat{F}(t)}{\hat{F}(t)},
$$

where $F(t)$ is the measured HXR flux and $\hat{F}(t)$ is the running average of $F(t)$. The red curve in Figure 4a shows $\hat{F}(t)$ calculated with the averaging time $\delta t=120 \mathrm{~s}$. The normalised time series $S(t)$ is shown in Figure 4b. Our basic method to recognise quasi-periodic sequences is the following. We measured time-intervals $P_{i}$ between successive HXR peaks and calculated the period $P=\left\langle P_{i}\right\rangle$ and its standard (r.m.s.) deviation $\sigma(P)$. Our criterion for a quasiperiodicity is $\sigma(P) / P \ll 1$. The values of $P$ and $\sigma(P)$ for different parts of the impulsive phase of the investigated flare are given in Table 1 .

Figures 3 and 4 together with Table 1 show the clear quasi-periodicity of the HXR pulses during the impulsive phase rise (time interval A in Figure 4b). Table 1 also shows that the period $P$ is longer near the impulsive phase maximum than during the impulsive phase 
Figure 3 The Yohkoh hard $\mathrm{X}$-ray (HXR) light curves in four energy ranges.
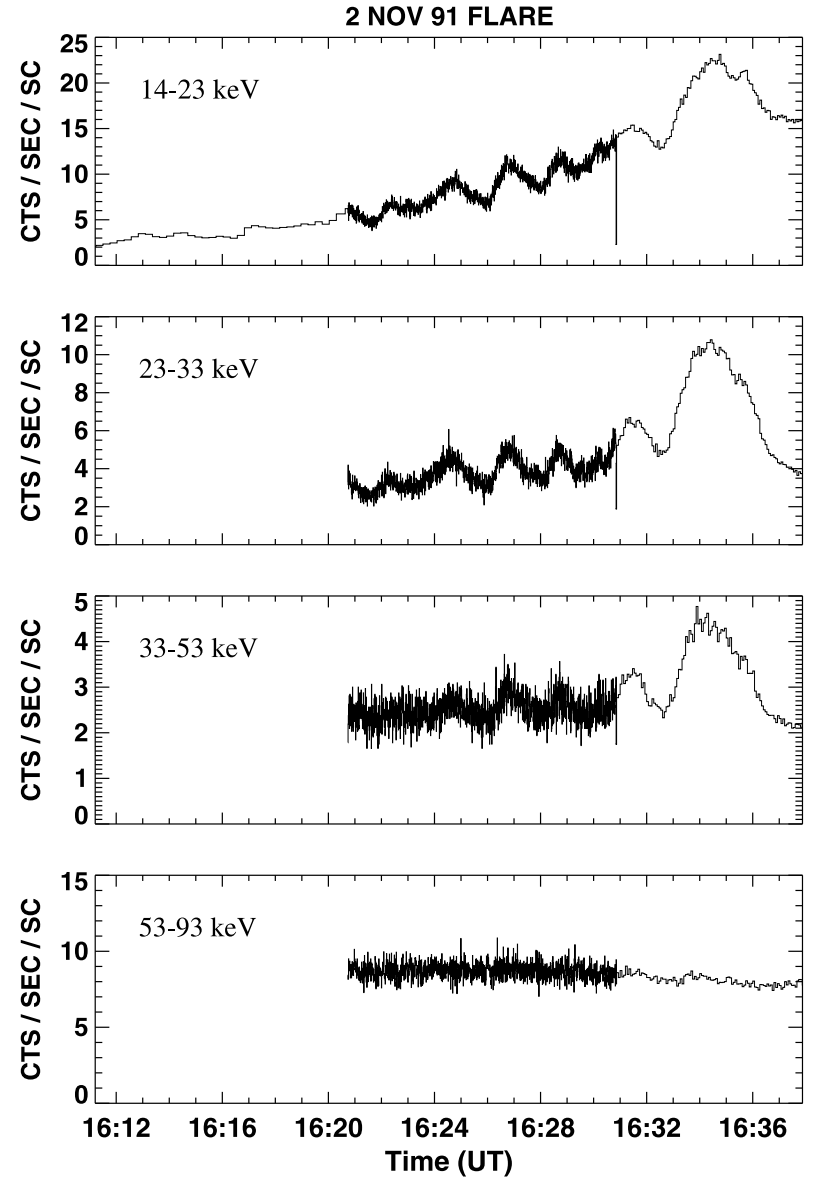

rise. This property was also observed in other flares (see Table 1 in Paper II). We interpret the variations of $P$ as caused by variations of the length of oscillating magnetic traps (see Sections 2.2 and 3).

Low-amplitude fluctuations are observed after the impulsive phase (time interval $\mathrm{B}$ in Figure $4 \mathrm{~b}$ ). Table 1 shows that these fluctuations also contain a weak quasi-periodic component.

The light curves in Figures 3 and 4 a can be divided into two components: the HXR pulses and a "quasi-smooth" component (emission below the pulses in the figures). The full amplitude Amp $S$ of the $S$-function for an HXR pulse is a measure of the ratio of the pulse intensity to the quasi-smooth component; the mean values of Amp $S$ are given in Table 1. We have explained the quasi-smooth component in Paper II as the result of superposed emission generated by many magnetic traps whose oscillations are shifted in phase.

\subsection{Investigation of the $\mathrm{X}$-ray Images}

X-ray images of the flare of 2 November 1991 are shown in Figures 5, 6, and 7. The color SXR images show a stable flaring loop. The bright central SXR source is the arcade channel, which in this flare was perpendicular to the plane of the images. 
Figure 4 (a) The Compton Gamma Ray Observatory HXR light curves. The red smoother curve is the running mean of the original light curve. (b) The normalised light curve $S(t)$ [see Equation (1)].

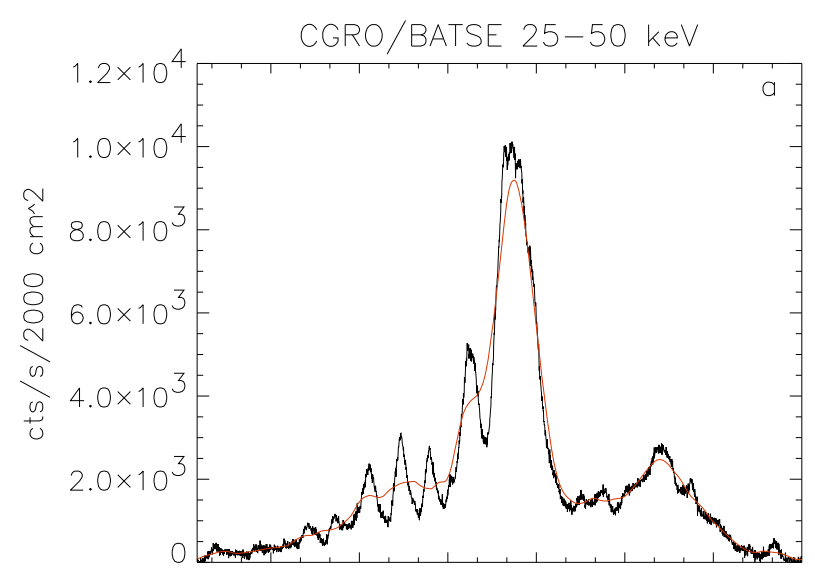

$16: 1816: 2416: 3016: 3616: 4216: 48$

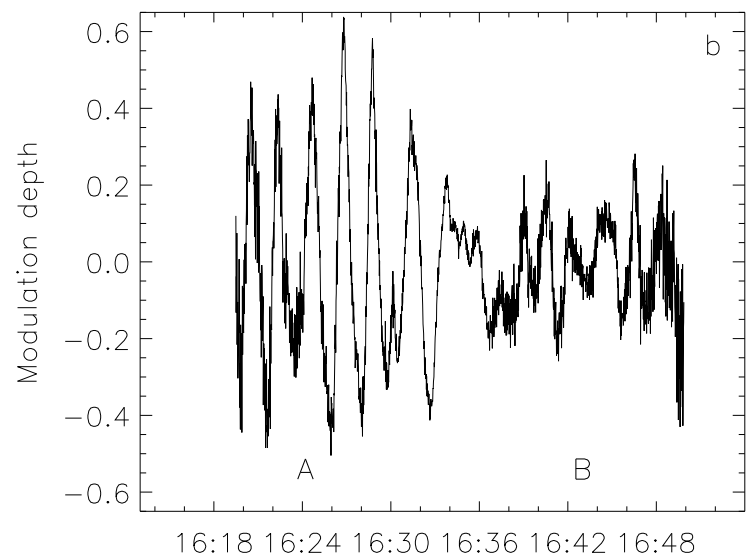

The HXR images are displayed as isocontours. The sensitivity of the Yohkoh Hard X-ray Telescope was moderate and the counting rates were low in this flare (see Figure 3); therefore it was necessary to apply a rather long integration time in the HXR image reconstruction.

Figure 5 shows a $14-23 \mathrm{keV}$ (channel L) image at the beginning of the sequence of HXR pulses. It shows that most of the $15 \mathrm{keV}$ electrons were confined within the loop-top source (no significant escape toward the footpoints).

The subsequent development of the HXR source can be better seen in the $23-33 \mathrm{keV}$ (channel M1) images. Figure 6 shows a sequence of the images. In Figure $6 \mathrm{c}$ we see a triangular (cusp) structure BPC, which is analogous to that in Figure 1. The source P is at the top of the cusp, and sources $\mathrm{B}$ and $\mathrm{C}$ are located at the places where the magnetic lines from the cusp meet the arcade channel. The B and C sources indicate that these magnetic lines and the magnetic field of the arcade channel are connected, which allows the precipitating electrons to penetrate into the dense plasma of the channel, emit HXRs, and heat the channel. Hence, sources B and C are the places where the magnetic traps are connected with the arcade channel (they are also the nodes of the trap oscillations). In Figures $6 \mathrm{~b}$ and $6 \mathrm{~d}$ the sources $\mathrm{B}$ and $\mathrm{P}$ are connected (not resolved).

The cusp is directed almost radially, with a slight inclination to the north. This radial direction of the cusp has been confirmed by late-phase SXR observations (see Figure 7). 
Figure 5 The Yohkoh soft X-ray image recorded with the Be119 filter (the colour image) and the 14-23 keV HXR image (isocontours) at the beginning of the sequence of HXR pulses. The isocontours are $0.20,0.39,0.59$, and $078 I_{\max }$, where $I_{\max }$ is the intensity of the brightest pixel.

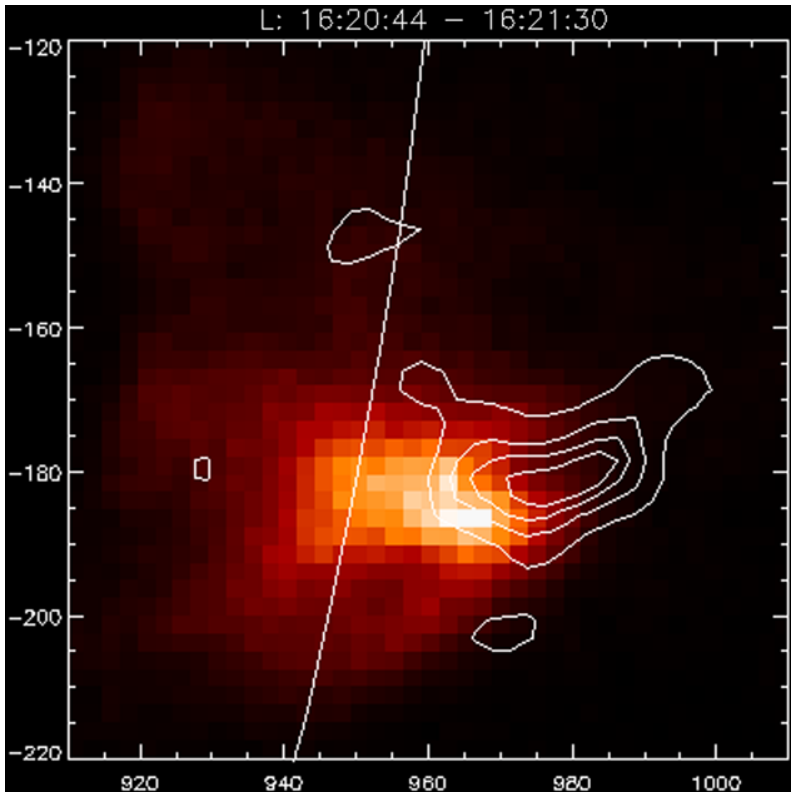

A specific feature of the present flare is that in most images in Figure 6 the HXR source $\mathrm{B}$ is much stronger than source $\mathrm{C}$. This indicates that the magnetic field within the cusp was asymmetric, which might have changed with time (see Section 3 for a discussion).

Figure 8 shows the structure of the magnetic field within the cusp (see Papers I and II). The structure is similar to that proposed by Aschwanden (2004a, 2004b) and Aschwanden et al. (1996). The magnetic reconnection is supposed to occur above the cusp $\mathrm{P}$ and the reconnection outflow excites the oscillations of the magnetic traps within the cusp.

Figure 6 also shows that the precipitation of the $25 \mathrm{keV}$ electrons toward the loop footpoints evolved in time. In Figures $6 a, 6 b, 6 d$, and $6 e$ the precipitation is weak (weak footpoint emission). In Figure $6 \mathrm{c}$ the sources near the footpoints are strong, which means strong precipitation. About 16:29 UT the footpoint source F began to grow in intensity and after 16:31 UT it was dominant in the M1 images (see Figure 6f), which means that the precipitation was very efficient. This indicates that strong chromospheric evaporation had been initiated. The changes of the precipitation of accelerated electrons from the BPC source are discussed in Section 3. In Figure 6f we also see a strong asymmetry in the $23-$ $33 \mathrm{keV}$ footpoint emission that is analogous to the asymmetry between the $\mathrm{B}$ and $\mathrm{C}$ sources. This is another result of the asymmetry in the precipitation of $25 \mathrm{keV}$ electrons from the cusp.

After 16:32 UT a strong increase in the SXR emission from the loop-top source occurred, which is seen as the saturation of the SXR images (Figure 9), and about 16:34-16:35 UT a strong peak was observed in the HXR light curves (Figures 3 and 4a). These two features clearly appeared because the dense plasma reached the loop-top and therefore a large number of electrons had been accelerated (see Section 3). These observations provide direct evidence that the strong HXR pulses are the result of the inflow of dense plasma that moves from the chromospheric evaporation into the loop-top source.

Figure 10 shows $14-23 \mathrm{keV}$ (channel L) images taken at 16:31-16:37 UT. The sources $\mathrm{B}$ and $\mathrm{P}$ are connected (not resolved) and remain strong in these images. The footpoint 

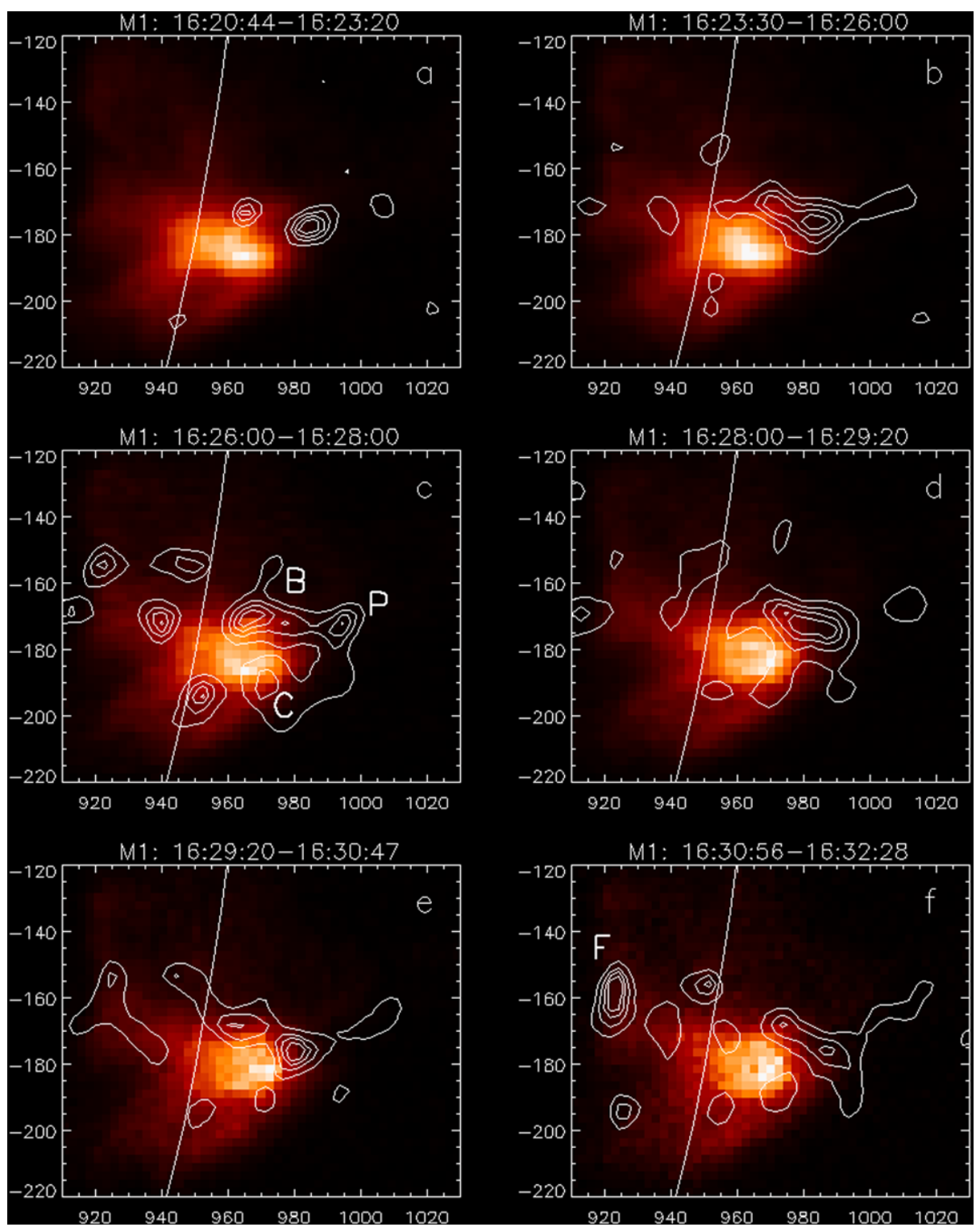

Figure 6 The sequence of SXR and $23-33 \mathrm{keV}$ HXR images for the time interval 16:20 - 16:32 UT.

source $\mathrm{F}$ is strong only in Figure 10c. An additional source A developed in the southern "leg" of the flaring loop. This indicates that after 16:32 UT many 15-keV electrons were precipitated into the loop leg, where they met dense plasma from the chromospheric evaporation.

In Section 3 we show that the observed properties of the loop-top source can be explained in terms of our model of oscillating magnetic traps. 
Figure 7 The late-phase SXR images of the flaring loop taken with the Al.1 filter. The cusp-like structure is clearly visible at the loop top.

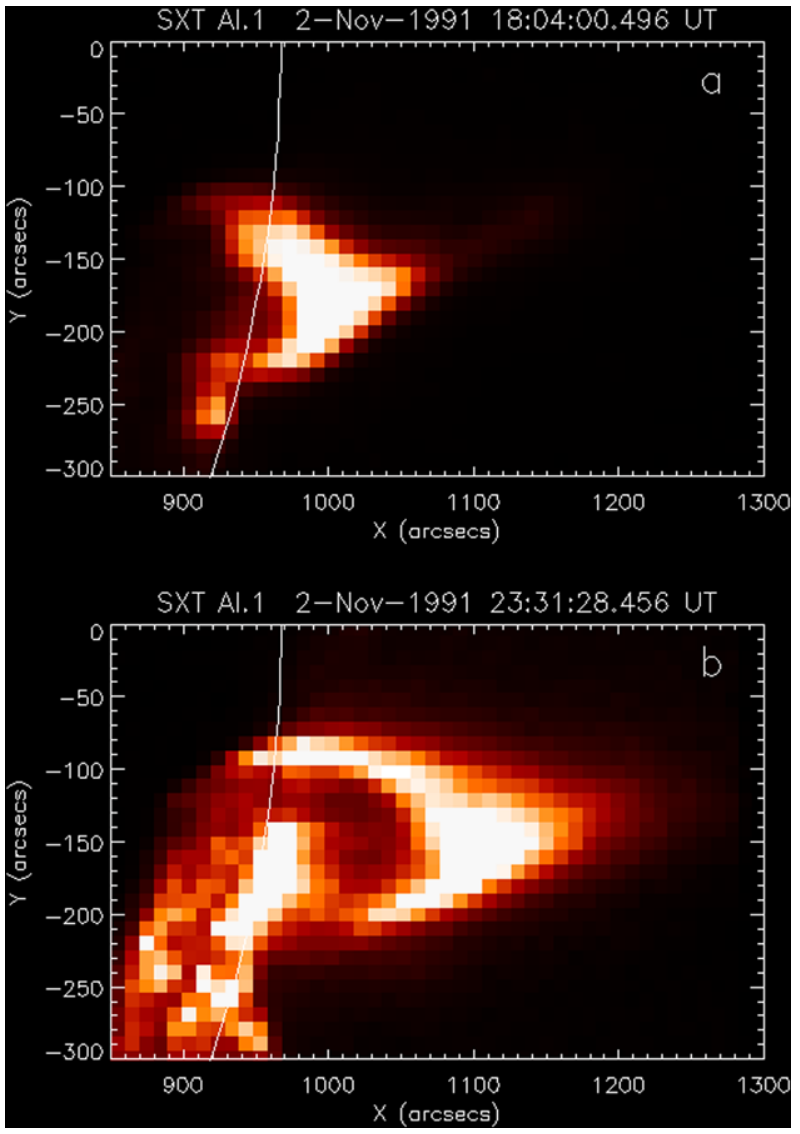

\section{Discussion}

In Papers I and II we developed the model of oscillating magnetic traps to explain the quasiperiodic oscillations of the HXR emission. This model is based on

i) The magnetic structure of the cusp according to Aschwanden et al. (1996) and Aschwanden (2004a).

ii) The mechanism of electron acceleration in magnetic traps during compression (Somov and Kosugi, 1997; Karlický and Kosugi, 2004).

iii) Our investigation of quasi-periodic variation in the HXR emission of solar flares (Papers I and II).

According to our model, the cusp (triangular) volume is filled with magnetic traps (see Figure 8). Magnetic reconnection at the top of the cusp (near P) generates the reconnection outflow, which excites the oscillations of the trap. Electrons are accelerated in the oscillating traps and emit hard X-rays.

The non-decaying sequences of the HXR pulses, like 16:20-16:32 UT in Figures 3 and 4, indicate that they are maintained by a feedback mechanism. Such a mechanism has been proposed in Paper II (the feedback between the amplitude of the magnetic-trap oscillations and the pressure of accelerated electrons). 
Figure 8 Magnetic structure of the BPC cusp. (The magnetic field $R$ in the flare region was perpendicular to the plane of the image; it was the arcade channel.) See text for details.

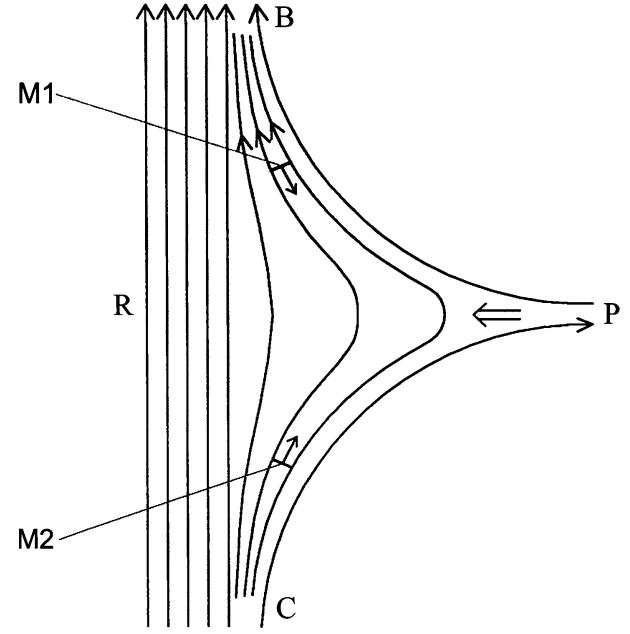

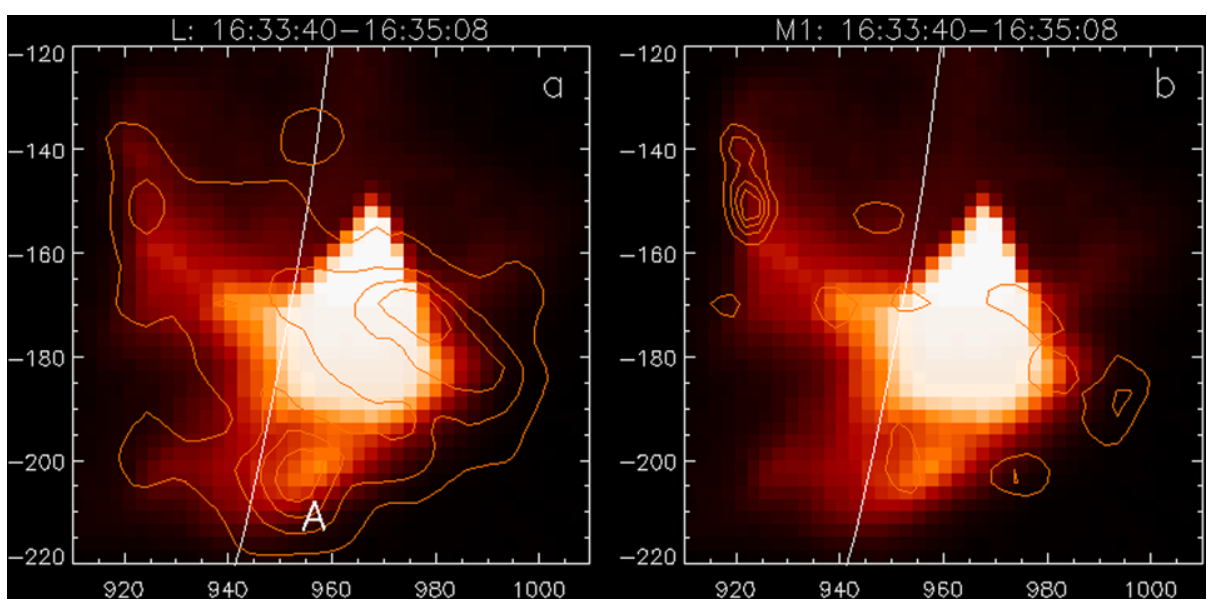

Figure 9 Yohkoh HXR images (contours) of (a) 14-23 keV taken at 16:33:40-16:35:08 UT and of (b) $23-33 \mathrm{keV}$ taken at 16:33:40 - 16:35:08 UT overlaid of the soft-X-ray image taken at 16:34:08 UT.

In Figure 6 we also see that the size of the BP source and the distribution of the HXR emission within the source changed with time. This is well explained by the model of oscillating magnetic traps because i) the BC magnetic mirrors are moving during the pulses (see Papers I and II), ii) the plasma distribution and the accelerated electrons within the traps change during the pulses.

Furthermore, the size and distribution of the emission in the $\mathrm{BC}$ source are more stable in the L-band images (see Figures 9 and 10). This is because $15 \mathrm{keV}$ electrons are generated in the traps, and their highest compression is weaker (higher $\chi_{\min }$ values, see below).

The sequences of the HXR images (Figures 6, 9, and 10) allowed us also to investigate the time variation of the precipitation of the accelerated electrons from the magnetic traps. The electron precipitation from a magnetic trap is controlled by the trap ratio, $\chi=B_{\max } / B_{\min }$, where $B_{\max }$ is the magnetic field strength at the mirrors B and C, and $B_{\min }$ is the strength at the middle of the trap. In the oscillating magnetic traps the parameter $\chi$ decreases during the 

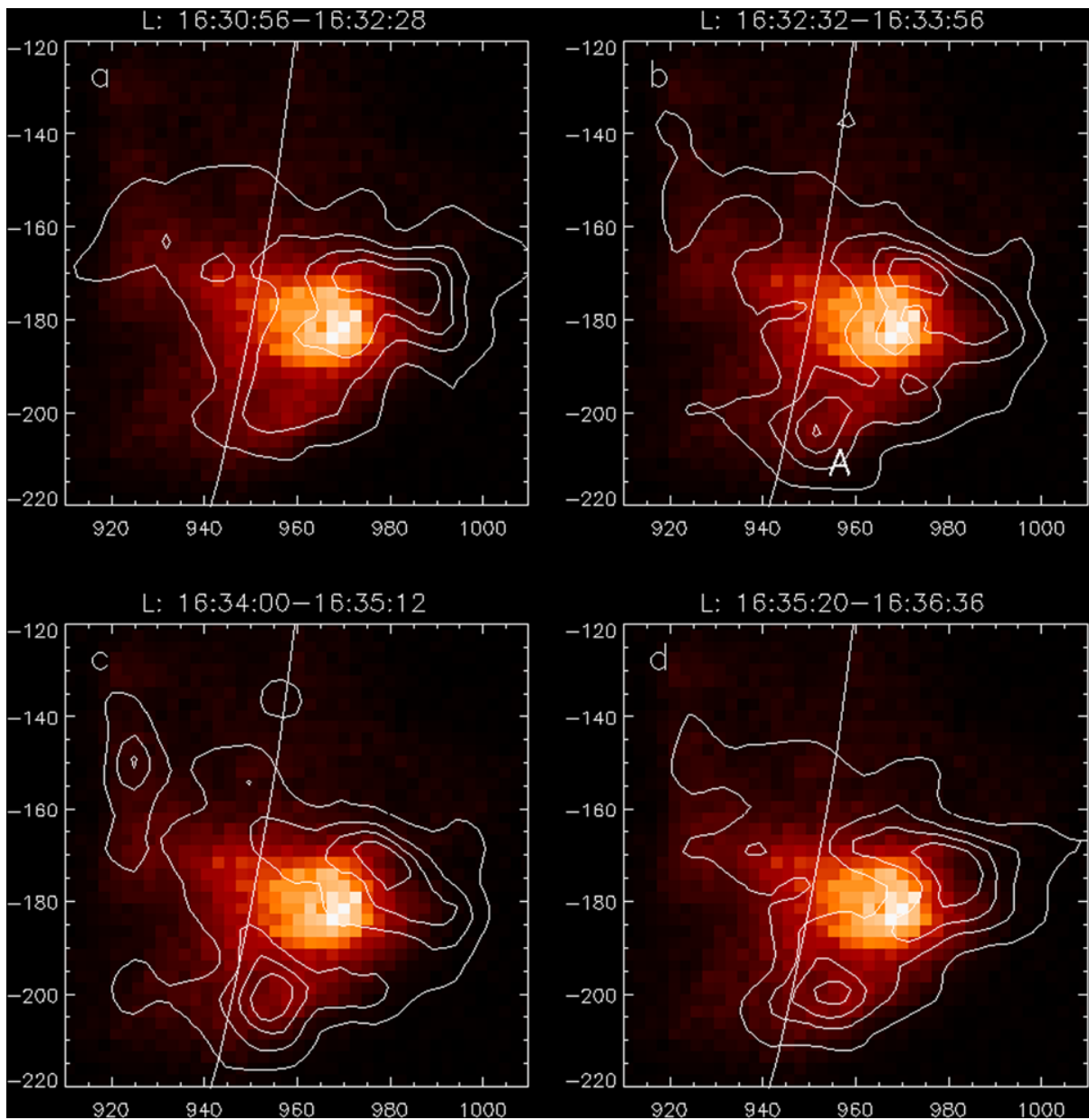

Figure 10 The sequence of 14-23 keV images for the time interval 16:30 - 16:36 UT. The same SXR image of 16:30:42 UT is displayed in all images to avoid saturated images.

compression of the traps and reaches its lowest value $\chi_{\min }$ during the highest compression, when the precipitation reaches its maximum.

The efficiency of the particle precipitation strongly depends on the value of $\chi_{\min }$. To illustrate this dependence we made some estimates under the simplifying assumption that the distribution of the accelerated electrons is isotropic. We describe the efficiency of precipitation by the ratio

$$
r=m / n
$$

where $m$ is the number $\left(\mathrm{s}^{-1}\right)$ of escaping electrons, and $n$ is the number $\left(\mathrm{s}^{-1}\right)$ of electrons that emit photons and are thermalised before they escape.

For the isotropic distribution

$$
r=\left(1-\cos \alpha_{\mathrm{c}}\right) / \cos \alpha_{\mathrm{c}},
$$


Table 2 The function $r\left(\chi_{\min }\right)$.

\begin{tabular}{lllllll}
\hline$\chi \min$ & 1 & 1.33 & 2 & 3 & 4 & 5 \\
$r$ & $\infty$ & 1.00 & 0.41 & 0.22 & 0.15 & 0.12 \\
\hline
\end{tabular}

$\chi_{\min }$ is the trap ratio at the highest compression.

$r=m / n$, where $m$ is the number $\left(\mathrm{s}^{-1}\right)$ of escaping electrons, $n$ is the number $\left(\mathrm{s}^{-1}\right)$ of electrons that emit photons and are thermalised before they escape.

where $\alpha_{\mathrm{c}}$ is the critical value of the pitch angle measured at the middle of the trap, such that electrons with $\alpha<\alpha_{\mathrm{c}}$ escape. The value of $\alpha_{\mathrm{c}}$ for the highest compression is related to $\chi_{\min }$ :

$$
\chi_{\min }=1 / \sin ^{2} \alpha_{\mathrm{c}} \text {. }
$$

Combining Equations (3) and (4), we obtain

$$
r=\frac{1}{\sqrt{1-1 / \chi_{\min }}}-1
$$

The function $r\left(\chi_{\min }\right)$ is given in Table 2. The table shows that when we observe strong precipitation $(r>1)$, this means a very strong compression of the traps $\left(\chi_{\min }<1.3\right)$.

Figure 6 and Table 2 indicate that during the sequence of pulses, 16:20-16:29 UT, the values of $\chi_{\min }$ for the $25 \mathrm{keV}$ electrons were $\chi_{\min }>3$ (weak electron precipitation) and after 16:31 UT they were $\chi_{\min }<1.3$ (strong precipitation). This shows that the dramatic change in the precipitation can be caused by an only moderate change in the value of $\chi_{\min }$.

According to our model of oscillating magnetic traps, the decrease in the value of $\chi_{\text {min }}$ in the sequence of pulses (i.e. increase in the highest compression) is due to the amplitude increase of the magnetic-trap oscillation. The amplitude gradually increases due to the feedback from the pressure pulses of the accelerated (non-thermal) electrons.

When the precipitation of $25 \mathrm{keV}$ electrons began to be strong (after 16:31 UT), the strong footpoint source $\mathrm{F}$ was observed in the M1 images (Figure 6). This shows that strong chromospheric evaporation began at that time. The dense plasma reached the loop top at about 16:32 UT, which caused i) the strong SXR emission seen as saturation in the SXR images (Figure 9), and ii) the strong HXR peak at about 16:34 UT (Figure 4a), which is due to the increase in the density in the oscillating magnetic traps. Figure $9 \mathrm{~b}$ shows that strong precipitation of $25 \mathrm{keV}$ electrons toward the footpoint F continued during the HXR maximum.

Further increase in the density inside the traps $\left(N \approx 10^{11} \mathrm{~cm}^{-3}\right)$ caused damping of electron acceleration when the collisional energy loss time $\tau_{\mathrm{c}}$ became similar to the acceleration time $\tau_{\text {acc }}$. This is the quick decrease in HXR emission after 16:36 UT (Figure 4a).

Another important feature of the flare is the strong asymmetry in electron precipitation (source B was much stronger than source C, and the north footpoint source F dominated over the south footpoint). This asymmetry is the result of the northward inclination of the cusp structure (see Figure 6c). Therefore the trap oscillations were asymmetric: the compression near source $B$ was stronger $\left(\chi_{\min }\right.$ lower) than near source $C$, hence the precipitation from $B$ was much stronger than from $C$.

The next property seen in the X-ray images (Figures 6, 9, and 10) is that after 16:31 UT the $25 \mathrm{keV}$ electrons (M1 images) could easily escape from source BP, but the precipitation of $15 \mathrm{keV}$ electrons (L images) remained low. This indicates that in the ensemble of the traps that generated the accelerated electrons, traps had different $\chi_{\min }$ values.

The comparison between the L and M1 images (Figures 6, 9, and 10) also shows that the BP source was systematically larger in the L images than in the M1 images. This shows that 
Figure 11 The relationship between size $d$ of the HXR loop-top source and period $P$ obtained in Paper I. The regression line was calculated for smaller flares $(P<60 \mathrm{~s})$. The red cross shows the position of the flare investigated in the present paper.

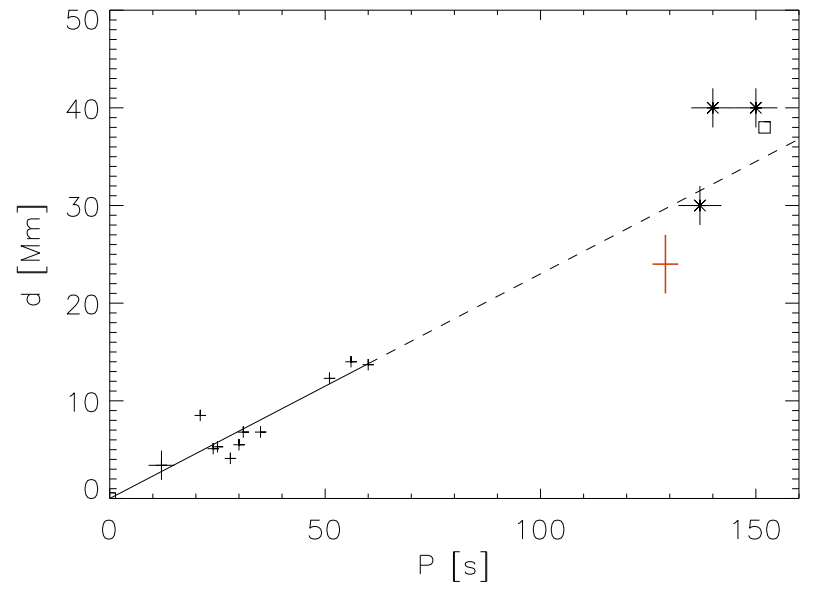

the traps that generated the $15 \mathrm{keV}$ electrons occupied a larger volume in the BP source than the traps that generated the $25 \mathrm{keV}$ electrons.

In Paper I we investigated the relationship between period and size of the HXR loop-top sources. Figure 11 shows the obtained correlation diagram. In that paper we described the size of the BPC cusp in large flares with a parameter $2 a$, where $a$ is the height of the triangle BPC. Therefore for the present flare we measured the value $a \approx 12 \mathrm{Mm}$ (Figure 6c) and plotted the point $(P, 2 a)$ as the red cross in Figure 11 .

Interestingly, in Figure 11 the points corresponding to the large $(P>120 \mathrm{~s})$ and small $(P<60 \mathrm{~s})$ flares are located near the same regression line. This can easily be explained by our model of oscillating magnetic traps.

i) In Paper I we have obtained the estimate

$$
d / P \approx(1 / \pi) v_{\mathrm{A}},
$$

where $v_{\mathrm{A}}$ is the Alfvén speed in the magnetic traps.

ii) In Paper I we have also found that during the impulsive phase the plasma pressure $p$ inside the traps reaches values that are of the order of the magnetic pressure

$$
p \approx B^{2} /(8 \pi)
$$

(see Section 3 in Paper I).

Using the relationships

$$
\begin{gathered}
p=2 N k T, \\
v_{\mathrm{A}}=B / \sqrt{4 \pi \rho},
\end{gathered}
$$

where $N$ is the electron number density, $k$ is the Boltzmann constant, and $\rho$ is the plasma density, we obtain

$$
v_{\mathrm{A}} \approx 1.8 \times 10^{4} \sqrt{T}\left[\mathrm{~cm} \mathrm{~s}^{-1}\right] .
$$

From Equations (6) and (10) we obtain

$$
d / P \approx 5.7 \times 10^{3} \sqrt{T}\left[\mathrm{~cm} \mathrm{~s}^{-1}\right] .
$$

Hence the points corresponding to large and small flares are located near the same regression line because 
Figure 12 Time variation of the temperature $T_{\mathrm{GOES}}$ derived from the GOES observations.

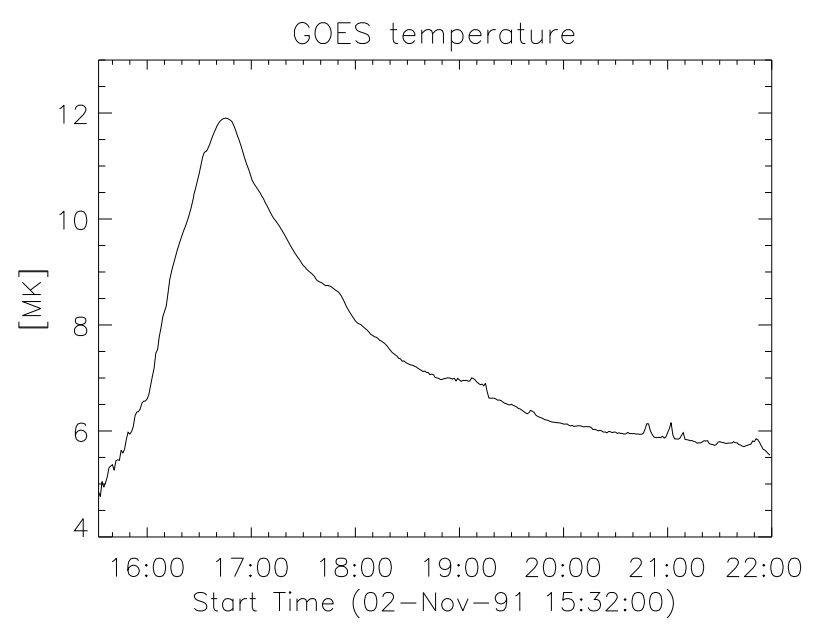

i) the gas pressure $p$ inside the magnetic traps is proportional to the magnetic pressure [Equation (7)], and

ii) the temperatures reached in these flares are similar [see Equation (11)].

We also verified whether Equation (11) provides a good approximation of the $d / P$ ratio. To do this, we estimated the temperature $T$ and the electron number density $N$ in the BP source from the SXR images that were recorded with the Be119 and Al.1 filters. This diagnostics was only possible for the time interval 16:20 - 16:30 UT, because later images were seriously saturated (see Figure 9). Using the filter ratio method, we found that the temperature slowly increased from 11 to $12 \mathrm{MK}$ and the electron number density was about $2 \times 10^{10} \mathrm{~cm}^{-3}$ during this time interval.

It has been shown (Jakimiec and Bak-Steślicka, 2011) that the temperature $T_{\mathrm{GOES}}$ estimated from the standard GOES records adequately represents the temperature of the hot plasma that generates the investigated SXR emission. The obtained values of $T_{\mathrm{GOES}}$ are shown in Figure 12. We see that during the impulsive phase the temperature reached $12 \mathrm{MK}$, which confirms that the flare was not very hot. This agrees with the temperatures $T_{\mathrm{GOES}}$ obtained for other "slow-LDE" flares (Bak-Steślicka and Jakimiec, 2005).

Inserting the value $T=12 \mathrm{MK}$ into Equation (11), we obtain $d / P \approx 0.20 \mathrm{Mm} \mathrm{s}^{-1}$, which agrees well with the value obtained from the observations (Figure 11), $d / P=$ $0.18 \pm 0.04 \mathrm{Mm} \mathrm{s}^{-1}$. Hence Equation (11) adequately describes the investigated flare and it probably provides a good approximation for other moderately hot flares.

Figure 7 shows late-phase SXR images of the investigated flare. These images are very important to confirm the cusp-like magnetic structure of the flare. A slow rise of the cusp structure is seen with the velocity about $4 \mathrm{~km} \mathrm{~s}^{-1}$.

\section{Summary}

The flare investigated in the present paper is exceptional, because

i) it was a large slowly developing flare located at the solar limb,

ii) it showed a clear sequence of quasi-periodic HXR pulses, and

iii) the available X-ray observations of the flare allowed us to investigate the development of its impulsive phase in some details. 
Our main aim was to show that the observations of the quasi-periodic oscillations in the HXR emission contain important information about the electron acceleration.

The analysis of the HXR observations has confirmed that i) quasi-periodic oscillations of the magnetic traps occur in the cusp-like magnetic structure and ii) the oscillations accelerate the electrons that generate the quasi-periodic HXR pulses.

The analysis of the observations allowed us also to investigate changes in the precipitation efficiency of the accelerated electrons during the impulsive phase. Moreover, we found that the precipitation efficiency (i.e. $\chi_{\min }$ ) is different in the different traps that participate in generating the HXR pulses.

Other important findings are that

i) The HXR pulses increase their amplitudes due to the amplitude increase of magnetictrap oscillations and the density increase within the traps caused by the chromospheric evaporation upflow.

ii) Accelerated electrons can precipitate into the arcade channel.

iii) The amplitude increase of the HXR pulses terminates when progressive density increase inside the traps inhibits the acceleration of electrons.

Generally, the model of oscillating magnetic traps is able to explain all the properties that were derived from the analysis of the HXR emission. In particular, the model explains i) the time variation of the electron precipitation observed in the sequences of the HXR images, ii) the strong asymmetry in the precipitation of accelerated electrons, and iii) the systematic differences in the precipitation of 15 and $25 \mathrm{keV}$ electrons revealed by comparing the $\mathrm{L}$ and M1 images. All this means that the HXR observations together with the model of oscillating magnetic traps give us a consistent picture of the development of the flare impulsive phase.

Acknowledgements The Yohkoh satellite is a project of the Institute of Space and Astronautical Science of Japan. The Compton Gamma Ray Observatory is a project of NASA. The authors are very grateful to the anonymous referee for her/his important remarks, which helped to improve this paper. We acknowledge financial support from the Polish National Science Centre grant 2011/03/B/ST9/00104.

Open Access This article is distributed under the terms of the Creative Commons Attribution License which permits any use, distribution, and reproduction in any medium, provided the original author(s) and the source are credited.

\section{References}

Aschwanden, M.J.: 2004a, Physics of the Solar Corona. An Introduction, Springer, Berlin, 536.

Aschwanden, M.J.: 2004b, Astrophys. J. 608, 554.

Aschwanden, M.J., Wills, M.J., Hudson, H.S., Kosugi, T., Schwartz, R.A.: 1996, Astrophys. J. 468, 398.

Bak-Steślicka, U., Jakimiec, J.: 2005, Solar Phys. 231, 95.

Fishman, G.J., Meegan, C.A., Wilson, R.B., Paciesas, W.S., Pendleton, G.N.: 1992, In: Shrader, C.R., Gehrels, N., Dennis, B. (eds.) The Compton Observatory Science Workshop, NASA CP-3137, 26.

Hudson, H.S., McKenzie, D.E.: 2000, In: Ramaty, R., Mandzhavidze, N. (eds.) High-Energy Solar Physics: Anticipating HESSI, ASP Conf. Ser. 206, 221.

Jakimiec, J., Bak-Steślicka, U.: 2011, Solar Phys. 272, 91.

Jakimiec, J., Tomczak, M.: 2010, Solar Phys. 261, 233 (Paper I).

Jakimiec, J., Tomczak, M.: 2012, Solar Phys. 278, 393 (Paper II).

Karlický, M., Kosugi, T.: 2004, Astron. Astrophys. 419, 1159.

Kosugi, T., Makishima, K., Murakami, T., Sakao, T., Dotani, T., Inda, M., et al.: 1991, Solar Phys. $136,17$.

Lipa, B.: 1978, Solar Phys. 57, 191.

Nakariakov, V.M., Melnikov, V.F.: 2009, Space Sci. Rev. 149, 119.

Somov, B.V., Kosugi, T.: 1997, Astrophys. J. 485, 859. 